\title{
Intramolecular Energy and Electron Transfer within a Diazaperopyrenium-Based Cyclophane
}

\author{
Xirui Gong, ${ }^{\dagger, \#}$ Ryan M. Young, ${ }^{\dagger, \ddagger, \# \odot ~ K a r e l ~ J . ~ H a r t l i e b, ~}{ }^{\dagger}$ Claire Miller, ${ }^{\dagger,}$ Yilei Wu, ${ }^{\dagger, \ddagger}$ Hai Xiao, ${ }^{\S}$ \\ Peng Li, ${ }^{\dagger}$ Nema Hafezi, ${ }^{\dagger}$ Jiawang Zhou, ${ }^{\dagger, f_{0}}$ Lin Ma, ${ }^{\dagger, \dagger}$ Tao Cheng, ${ }^{\S}{ }^{\circ}$ William A. Goddard, III, ${ }^{\S}$

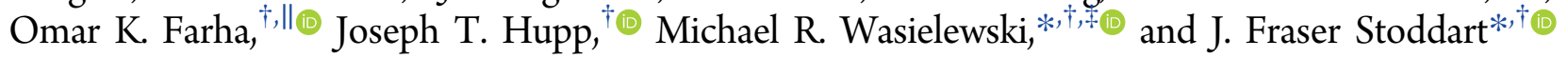

${ }^{\dagger}$ Department of Chemistry and ${ }^{\ddagger}$ Argonne-Northwestern Solar Energy Research (ANSER) Center, Northwestern University, 2145 Sheridan Road, Evanston, Illinois 60208, United Sates

"Department of Chemistry, Faculty of Science, King Abdulaziz University, Jeddah 22254, Saudi Arabia

${ }^{\S}$ Materials and Process Simulation Center, California Institute of Technology, Pasadena, California 91125, United States

Supporting Information

ABSTRACT: Molecules capable of performing highly efficient energy transfer and ultrafast photoinduced electron transfer in well-defined multichromophoric structures are indispensable to the development of artificial photofunctional systems. Herein, we report on the synthesis, characterization, and photophysical properties of a rationally designed multichromophoric tetracationic cyclophane, DAPPBox ${ }^{4+}$, containing a diazaperopyrenium $\left(\mathrm{DAPP}^{2+}\right)$ unit and an extended viologen $\left(\mathrm{ExBIPY}^{2+}\right)$ unit, which are linked together by two $p$-xylylene bridges. Both ${ }^{1} \mathrm{H}$ NMR spectroscopy and single-crystal X-ray diffraction analysis confirm the formation of an asymmetric, rigid, box-like cyclophane, DAPPBox ${ }^{4+}$. The solid-state superstructure of this cyclophane reveals a herringbone-type packing motif, leading to two types of $\pi \cdots \pi$ interactions: (i) between the ExBIPY ${ }^{2+}$ unit and the $\mathrm{DAPP}^{2+}$ unit $(\pi \cdots \pi$ distance of $3.7 \AA)$ in the adjacent parallel cyclophane, as well as (ii) between the ExBIPY ${ }^{2+}$ unit $(\pi \cdots \pi$ distance of $3.2 \AA)$ and

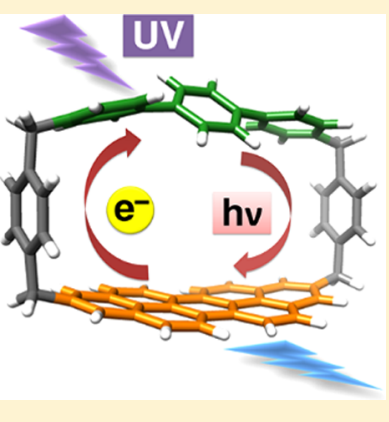
phenylene ring in the closest orthogonal cyclophane. Moreover, the solution-phase photophysical properties of this cyclophane have been investigated by both steady-state and time-resolved absorption and emission spectroscopies. Upon photoexcitation of DAPPBox $^{4+}$ at $330 \mathrm{~nm}$, rapid and quantitative intramolecular energy transfer occurs from the ${ }^{1 *}$ ExBIPY $^{2+}$ unit to the DAPP ${ }^{2+}$ unit in 0.5 ps to yield ${ }^{1 *} \mathrm{DAPP}^{2+}$. The same excitation wavelength simultaneously populates a higher excited state of ${ }^{1 *} \mathrm{DAPP}{ }^{2+}$ which then undergoes ultrafast intramolecular electron transfer from ${ }^{1 *} \mathrm{DAPP}^{2+}$ to $\mathrm{ExBIPY}^{2+}$ to yield the $\mathrm{DAPP}{ }^{3+\bullet}-\mathrm{ExBIPY}^{+\bullet}$ radical ion pair in $\tau=1.5 \mathrm{ps}$. Selective excitation of $\mathrm{DAPP}^{2+}$ at $505 \mathrm{~nm}$ populates a lower excited state where electron transfer is kinetically unfavorable.

\section{INTRODUCTION}

The construction of well-organized multichromophoric architectures, capable of performing efficient energy transfer (EnT) and electron transfer ${ }^{1}$ (ET), is an indispensable part of the development of photofunctional systems. The key to the design of such synthetic systems is controlling the electronic interactions between donors (D) and acceptors (A) to optimize efficient EnT and /or ET. Encouraging progress has been made on the construction of photofunctional systems, extending from discrete (1D) to extended (2D and 3D) structures, such as $\pi$-stacked molecules, ${ }^{2}$ covalently linked porphyrin arrays, ${ }^{3}$ dendrimers, ${ }^{4}$ chromophoric polymers, ${ }^{5}$ self-assembled structures, ${ }^{6}$ and hybrid organic-inorganic structures. ${ }^{7}$ Recently, the photophysical properties (either EnT or ET) of a newly synthesized tetracationic cyclophane, ExBox ${ }^{4+}$, and its supramolecular complexes have been investigated. ${ }^{8}$ ExBox $^{4+}$ is related to cyclobis(paraquat-p-phenylene) wherein the $4,4^{\prime}$ bipyridinium units have been replaced with an extended p-phenylene-bridged bipyridinium unit, ExBIPY ${ }^{2+}$. Multielectron accumulation ${ }^{7 \mathrm{e}}$ and photoinduced charge shift reactions of $\mathbf{E x B o x}^{4+}$ have been demonstrated by intramolecular ${ }^{9}$ through-bond electron transfer from the $p$-xylylene bridges to the extended bipyridinium units $\left(\mathrm{ExBIPY}^{2+}\right)$ within ExBox ${ }^{4+}$ itself as well as by intermolecular ${ }^{6 c}$ photoinduced charge shift within perylene $C \mathrm{ExBox}^{4+}$ complexes. On the other hand, EnT processes have been observed $^{6, f}$ in supramolecular systems, by binding ExBox $^{4+}$ to other chromophores, like perylene3,4:9,10-bis(dicarboximide)s (PDIs) (Figure 1a). Although one of the $\mathrm{ExBIPY}^{2+}$ units in ExBox ${ }^{4+}$ can undergo intramolecular photoinduced charge separation if excited with UV light, optimum utilization of the solar spectrum for artificial photosynthesis requires systems that absorb at wavelengths $>400 \mathrm{~nm}$. Given this requirement, we have designed an asymmetric cyclophane which incorporates one $\mathrm{ExBIPY}^{2+}$ (Figure 1b) electron acceptor unit. For a second chromophore to work alongside an $\mathrm{ExBIPY}^{2+}$ unit within the geometry of a cyclophane molecule, that chromophore must satisfy several criteria. First of all, the chromophore must be able to form a donor-acceptor pair with an ExBIPY ${ }^{2+}$ unit. Second, it should have a similar

Received: December 31, 2016

Published: February 22, 2017 
(a)

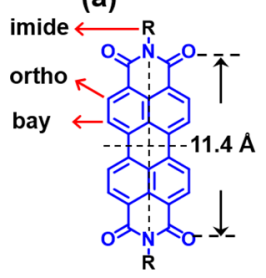

PDI (b)

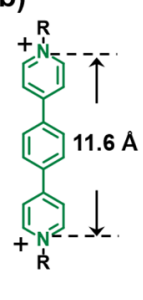

(c)

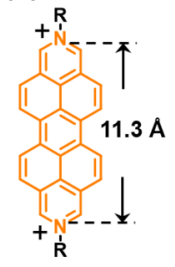

DAPP ${ }^{2+}$ (d)

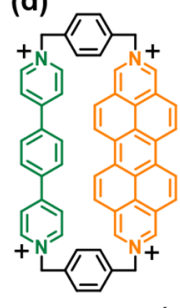

Figure 1. Structural formulas of (a) PDI, (b) $\mathrm{ExBIPY}^{2+}$, (c) DAPP $^{2+}$ and (d) DAPPBox ${ }^{4+}$.

length as an $\mathrm{ExBIPY}^{2+}$ unit to ensure that the cyclophane retains its rectangular shape, which (i) promotes good electronic coupling between the donor and acceptor in the face-toface geometry as well as (ii) provides an opportunity to bind guests, which may be used to modulate the coupling between the donor and the acceptor, thereby allowing control over charge separation and recombination rates. ${ }^{6 c}$

A class of molecules that match the above criteria are the diazaperopyrenium dications, ${ }^{10}$ shown in Figure 1c. Although these dications are structurally related and share optical properties similar to those of the PDIs, they possess enhanced solubility as a result of their dicationic nature. Additionally, $\mathrm{DAPP}^{2+}$ dications can be dissolved selectively either in organic solvents (soft counterions, e.g., $\mathrm{PF}_{6}^{-}, \mathrm{BF}_{4}{ }^{-}$) or in aqueous environments (hard counterions, e.g., $\mathrm{CF}_{3} \mathrm{CO}_{2}^{-}, \mathrm{Cl}^{-}$). Since energy transfer between $\mathrm{ExBIPY}^{2+}$ and PDI has been described $^{6 e, f}$ in self-assembled systems, we expect that, by incorporating a $\mathrm{DAPP}^{2+}$ dicationic unit into a constitutionally asymmetric cyclophane along with an $\mathrm{ExBIPY}^{2+}$ unit, the unique arrangement of multichromophoric units could demonstrate special photophysical properties, while avoiding solubility issues commonly associated with PDIs. ${ }^{11}$ The known properties ${ }^{10 \mathrm{e}}$ of the $\mathrm{DAPP}^{2+}$ dication suggest these dicationic units are suitable substitutes for PDIs for incorporation into multichromophoric cyclophanes.

Here, we report a rational design and preparation of a cyclophane, DAPPBox ${ }^{4+}$ (Figure 1d), containing one DAPP ${ }^{2+}$ unit and one ExBIPY ${ }^{2+}$ unit, which are bridged together by two $p$-xylylene linkers. This constitutionally asymmetric tetracationic cyclophane is readily soluble in both organic solvents, e.g., $\mathrm{MeCN}, \mathrm{MeNO}_{2}$, and $\mathrm{DMF}$, as a $\mathrm{PF}_{6}{ }^{-}$salt, and in water as a

$\mathrm{Cl}^{-}$or $\mathrm{TFA}^{-}$salt. In this investigation, we have combined steady-state measurements, visible and near-infrared (NIR) transient absorption spectroscopy, and electronic structure calculations in order to investigate the photophysical processes within DAPPBox ${ }^{4+}$. Our results show that photoexcitation of the $\mathrm{ExBIPY}^{2+}$ electron acceptor within DAPPBox ${ }^{4+}$ results in ultrafast energy transfer to the lowest excited state of the $\mathrm{DAPP}^{2+}$ electron donor, followed by competitive fluorescence and intersystem crossing. Owing to the overlapping absorption of the subunits at $330 \mathrm{~nm}$, excitation at this wavelength also leads to a small population of highly excited ${ }^{1 *} \mathrm{DAPP}^{2+}$ which decays by electron transfer to $\mathrm{DAPP}^{3+\bullet}-\mathrm{ExBIPY}^{+}$in $\tau=1.5$ ps. Electron transfer from the lowest excited state of ${ }^{1}$ DAPP ${ }^{2+}$ around $2.46 \mathrm{eV}$ is kinetically unfavorable in the cyclophane, leading to the same decay previously observed in $\mathrm{DAPP}^{2+}$. This rationally designed DAPPBox ${ }^{4+}$ can perform both energy- and electron-transfer processes. The detailed study of the photophysical properties of the cyclophane constitutes an important fundamental step toward the understanding of competitive photophysical processes in the context of controlled molecular geometries.

\section{RESULTS AND DISCUSSION}

Synthesis of the DAPPBox $4 \mathrm{PF}_{6}$. The synthesis of DAPPBox $^{4+}$ is outlined in Scheme 1. Briefly, $S_{\mathrm{N}} 2$ reactions between an excess of 3,4,9,10-tetrakis (chloromethyl)perylene (1) and 4-(aminomethyl)benzenemethanol (2) in $\mathrm{Me}_{2} \mathrm{SO}$ at room temperature for $5 \mathrm{~h}$, lead to the formation of 3 . Subsequently, 3 is oxidized by 2,3-dichloro-5,6-dicyano-1,4-benzoquinone (DDQ) in DMF at room temperature for $8 \mathrm{~h}$ to afford, after counterion exchange, $4 \cdot 2 \mathrm{PF}_{6}$. Bromination of $4 \cdot 2 \mathrm{PF}_{6}$ with $\mathrm{PBr}_{3}$ in $\mathrm{MeCN}$ at room temperature overnight, followed by counterion exchange with $\mathrm{NH}_{4} \mathrm{PF}_{6}$, results in the fomation of the dibromide BrDAPP.2PF . Finally, pyrene-templated $^{8 \mathrm{~b}}$ cyclization between BrDAPP. $2 \mathrm{PF}_{6}$ and 4,4' $4^{\prime}$ (1,4-phenylene) bispyridine (ExBIPY) in MeCN at room temperature for 7 days, followed by precipitation of the crude product with tetrabutylammonium chloride (TBACl), gives pyreneCDAPPBox $4 \mathrm{Cl}$. This complex can then be dissolved in $\mathrm{H}_{2} \mathrm{O}$ and the aqueous solution subjected to continuous extraction with $\mathrm{CHCl}_{3}$ for 3 days in order to remove the pyrene guest molecule from the cyclophane. The desired product DAPPBox $4 \mathrm{PF}_{6}$ was isolated by reverse-phase column chromatography, followed by counterion

Scheme 1. Synthesis of DAPPBox $4 \mathrm{PF}_{6}$
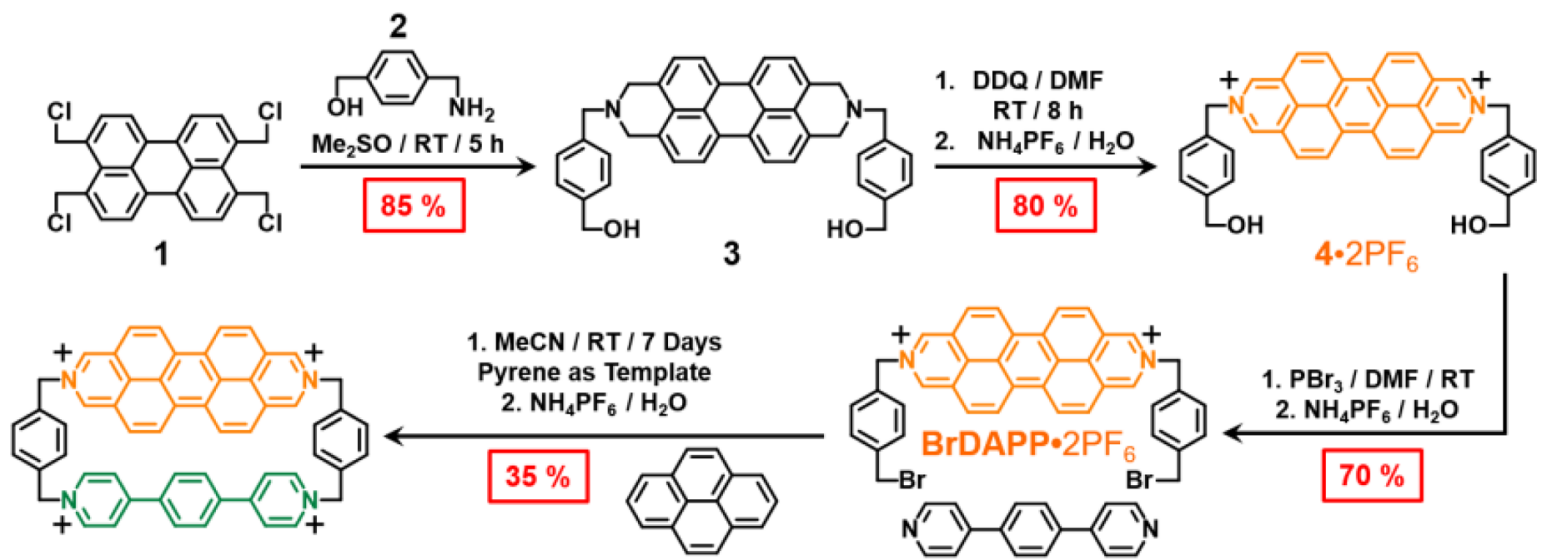

DAPPBox॰4PF
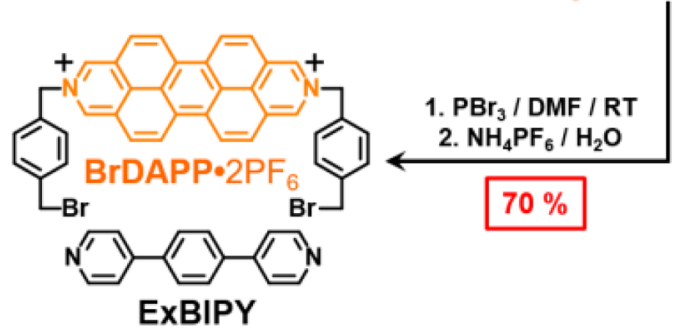

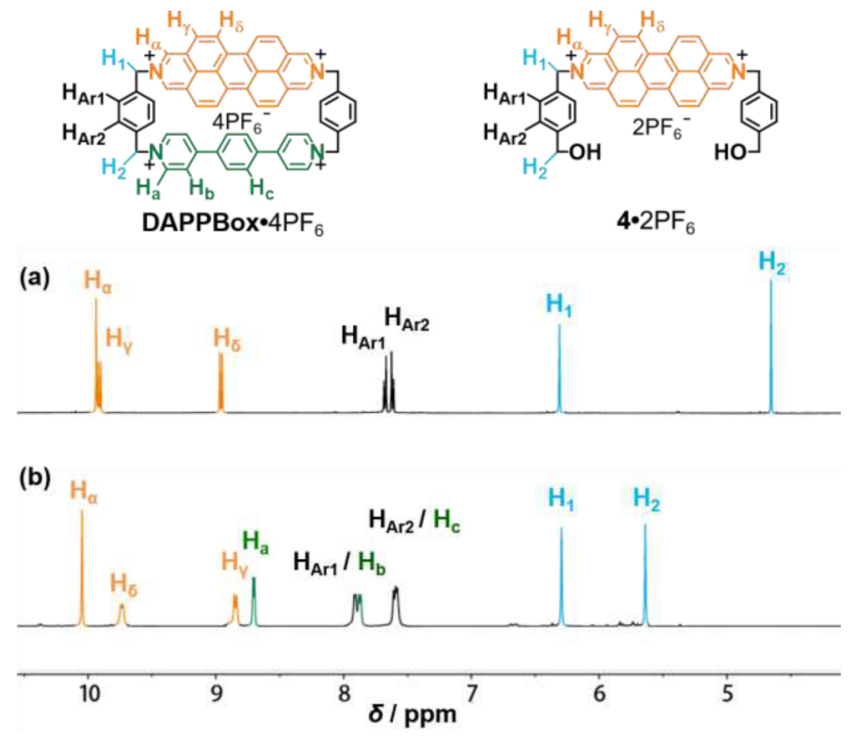

Figure 2. ${ }^{1} \mathrm{H}$ NMR spectra of (a) $4 \cdot 2 \mathrm{PF}_{6}$ and (b) DAPPBox $4 \mathrm{PF}_{6}$ $\left(500 \mathrm{MHz}, \mathrm{CD}_{3} \mathrm{CN}, 298 \mathrm{~K}\right)$.

exchange $\left(\mathrm{NH}_{4} \mathrm{PF}_{6} / \mathrm{H}_{2} \mathrm{O}\right)$ in $35 \%$ yield. The formation of DAPPBox $4 \mathrm{PF}_{6}$ was confirmed by recording the ${ }^{1} \mathrm{H}$ NMR spectrum (Figure 2) in $\mathrm{MeCN}$ at room temperature. The peaks in the spectrum were assigned on the basis of analyses of two-dimensional ${ }^{1} \mathrm{H}-{ }^{1} \mathrm{H}$ COSY NMR (Figure S1a) and 2D NOESY NMR (Figure S1b) spectra. As a result of its asymmetric constitution, two sets of peaks can be assigned to the methylene protons $\left(\mathrm{H}_{1} / \mathrm{H}_{2}, \delta=6.27 / 5.63 \mathrm{ppm}\right)$ as well as another two sets of peaks to the phenylene protons $\left(\mathrm{H}_{\mathrm{Ar} 1} / \mathrm{H}_{\mathrm{Ar} 2}, \delta=7.87 /\right.$ $7.59 \mathrm{ppm}$ ) of the $p$-xylylene linkers. High-resolution mass spectrometry (HRMS) revealed the presence of the $\left[\mathrm{M}-\mathrm{PF}_{6}\right]^{+}$, $\left[\mathrm{M}-2 \mathrm{PF}_{6}\right]^{2+}$, and $\left[\mathrm{M}-3 \mathrm{PF}_{6}\right]^{3+}$ species which were detected at $m / z=1203.2074,529.1266$, and 304.4293, respectively.

Further evidence for cyclophane formation comes from single-crystal X-ray diffraction (XRD), Figure 3. Single crystals were grown by vapor diffusion of $i-\mathrm{Pr}_{2} \mathrm{O}$ into an $\mathrm{MeCN}$ solution of DAPPBox-4TFA during 1 week. The solid-state structure (Figure 3a) reveals an asymmetric, box-like cyclophane, with average dimensions of $14.1 \AA \times 7.1 \AA$ (Figure $3 \mathrm{~b}$ ). The two torsional angles between the pyridinium and the $p$-phenylene rings are $\sim 17^{\circ}$ and $\sim 20^{\circ}$ (Figure $3 \mathrm{c}$ ), respectively; i.e., they are smaller than the average torsional angle $\left(\sim 30^{\circ}\right)^{8 \mathrm{a}}$ in $\operatorname{ExBox}^{4+}$. This flatter conformation for the ExBIPY ${ }^{2+}$ unit in DAPPBox $^{4+}$ is probably a consequence of intermolecular $\pi \cdots \pi$ interactions ${ }^{12}$ between the ExBIPY ${ }^{2+}$ unit and adjacent cyclophanes. The $p$-xylylene ring in the closest adjacent cyclophane $(\pi \cdots \pi$ distance of $3.2 \AA)$ is associated with a torsional angle of $\sim 17^{\circ}$ in the $\operatorname{ExBIPY}^{2+}$ unit, while the larger torsional angle $\left(\sim 20^{\circ}\right)$ is adjacent to the relative weaker intermolecular aromatic recognition $(\pi \cdots \pi$ distance of $3.7 \AA)$ between the $\mathrm{ExBIPY}^{2+}$ unit and the $\mathrm{DAPP}^{2+}$ unit in another adjacent cyclophane. The perspective view of the solid-state superstructure (Figure $3 \mathrm{~d}$ ) of DAPPBox $^{4+}$ reveals only partial $\pi \cdots \pi$ overlap between the $\mathrm{ExBIPY}^{2+}$ unit and a $\mathrm{DAPP}^{2+}$ unit in a neighboring cyclophane, most likely arising from the balance between Coulombic repulsions and the maximization of $\pi$-overlap. ${ }^{10 \mathrm{~h}}$ The overall (a)
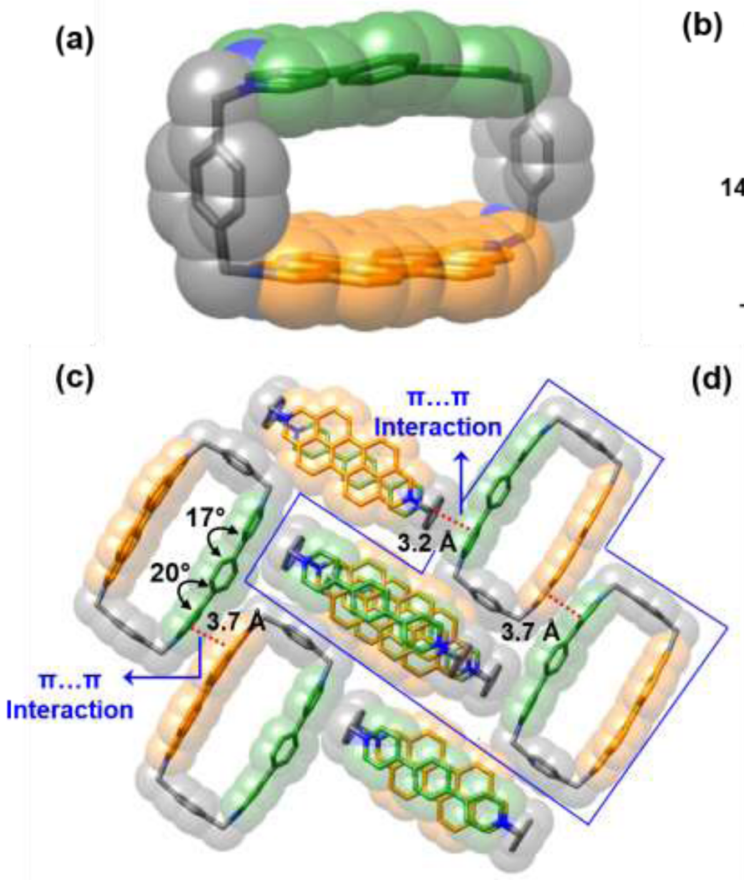

(b)

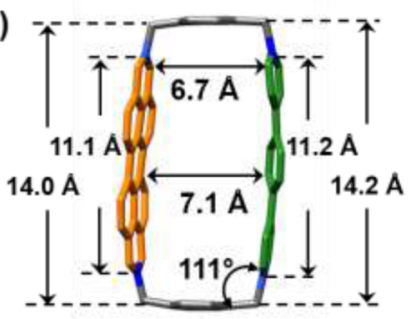

(d)

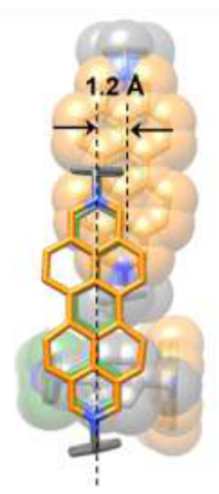

Figure 3. Solid-state (super)structure of the DAPPBox ${ }^{4+}$ obtained from X-ray crystallography on single crystals of DAPPBox 4 TFFA. (a) Perspective view of the DAPPBox ${ }^{4+}$ as a stick representation with the corresponding semitransparent space-filling representations superimposed upon it. (b) Plan view of stick representation DAPPBox ${ }^{4+}$ ring showing distances and torsional angles associated with the ring's geometry. (c) Solid-state superstructure of DAPPBox ${ }^{4+}$ revealing a herringbone type of packing and two types of $\pi \cdots \pi$ interactions ${ }^{12}$ as labeled above, resulting in flatter features for the ExBIPY ${ }^{2+}$ unit (smaller torsional angle between adjacent pyridinium rings, $\sim 17^{\circ}$ and $\sim 20^{\circ}$, respectively) in DAPPBox ${ }^{4+}$, compared ${ }^{8 a}$ $\left(\sim 30^{\circ}\right)$ with its ExBox ${ }^{4+}$ analogue. (d) Perspective view of the superstructure of DAPPBox ${ }^{4+}$ demonstrating the partial overlap between the ExBIPY ${ }^{2+}$ and $\mathrm{DAPP}^{2+}$ units in adjacent cyclophanes, probably resulting from the balance between Coulombic repulsions and the maximization of $\pi$-overlap. 
superstructure of $\mathrm{DAPPBox}^{4+}$ reveals a herringbone type of packing.

The templating role played by pyrene during the synthesis of the cyclophane is supported by the solid-state superstructure (Figure S5) of the complex, pyrene CDAPPBox $\cdot 4 \mathrm{PF}_{6}$. Single crystals, suitable for X-ray crystallography, were obtained by slow vapor diffusion of $i \mathrm{Pr}_{2} \mathrm{O}$ into a $\mathrm{MeNO}_{2}$ solution of the complex. The superstructure reveals that pyrene sits inside the box at distances of $3.4 \AA$ from the $\mathrm{DAPP}^{2+}$ plane and $3.6 \AA$ from the center of the ExBIPY ${ }^{2+}$ unit. For full details, see the Supporting Information. In addition, the characteristic ${ }^{1} \mathrm{H}$ NMR signals of the macrocycle shift upon addition of pyrene (Figure S2), proving that complexation occurs between pyrene and DAPPBox ${ }^{4+}$ in solution. A UV/vis absorption-based Job plot (Figure S3b) confirms the 1:1 stoichiometry between DAPPBox $^{4+}$ and pyrene in $\mathrm{MeCN}$, an observation which is in agreement with the solid-state superstructure. Furthermore, the fluorescence titration (Figure S3c) carried out upon the addition of pyrene into an MeCN solution of the DAPPBox ${ }^{4+}$ (excitation at $440 \mathrm{~nm}$ ) shows a decrease of $\mathrm{DAPP}^{2+}$ fluorescence $(515 \mathrm{~nm})$, accompanied by a slight blue shift of the emission maxima, caused by either electronic perturbation or structural distortion upon host-guest formation. Based on fluorescence titration data, the binding affinity $K_{\mathrm{a}}$ of complexation was calculated $^{11 \mathrm{~b}}$ (Figure S3d) to be $(3.73 \pm 0.35) \times 10^{5} \mathrm{M}^{-1}$.

Reference Compound Absorption and Emission. Two groups of reference compounds with methyl groups (Figure 4a) and benzyl groups (Figure 4b) attached to the nitrogen atoms in the 2 and 9 positions ${ }^{10 h}$ were investigated to understand the basic photophysical processes involved in each chromophore. Based on the results obtained from these reference compounds, we observed that the benzyl groups enable an ET deactivation pathway, a feature which was not found to be present in the corresponding methylated reference compound. ${ }^{9}$ Thus, the photophysics of the methylated reference compounds $\left(\right.$ Me-ExBIPY $^{2+}$ and Me-DAPP ${ }^{2+}$ ) were compared to that of DAPPBox $^{4+}$, when $330 \mathrm{~nm}$ photons were used to excite $\mathrm{ExBIPY}^{2+}$, while the photophysics of the benzylated reference compounds (Bn-ExBIPY ${ }^{2+}$ and Bn-DAPP ${ }^{2+}$ ) were compared to that of $\mathrm{DAPPBox}{ }^{4+}$, when $\mathrm{DAPP}^{2+}$ was selectively excited at 505 and $414 \mathrm{~nm}$. Our data show that, in $\mathrm{MeCN}$ at room temperature, Bn-ExBIPY ${ }^{2+}$ exhibits weak UV fluorescence (a)

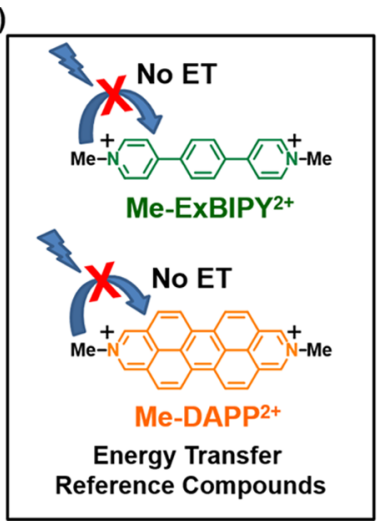

(b)

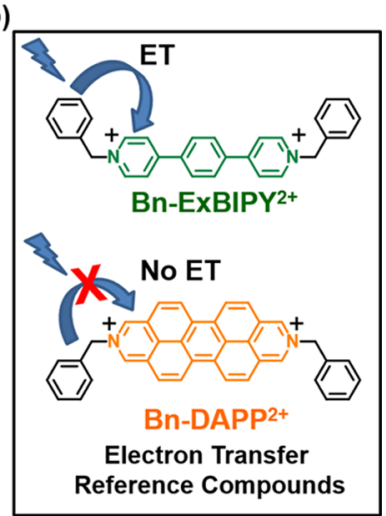

Figure 4. (a) Structural formulas of Me-ExBIPY ${ }^{2+}$ and Me-DAPP ${ }^{2+}$, which were used as reference compounds for the EnT process within DAPPBox $^{4+}$. (b) Structural formulas of Bn-ExBIPY ${ }^{2+}$ and BnDAPP $^{2+}$, which were used as reference compounds for the ET process within DAPPBox ${ }^{4+}$. $\left(\lambda_{\max }=378 \mathrm{~nm}, \Phi_{\mathrm{fl}}=3.8 \%\right)$, presumably as a result of deactivation by photoinduced electron transfer from the benzyl rings to ExBIPY $^{2+}$, , while strong UV emission is observed $\left(\lambda_{\max }=\right.$ $364 \mathrm{~nm}, \tau=1.56 \mathrm{~ns}, \Phi_{\mathrm{fl}}=69 \%$, Figure S10) in the case of Me-ExBIPY ${ }^{2+}$, where the electron-transfer pathway is not present. Conversely, neither methyl nor benzyl substituents significantly affect the $\mathrm{DAPP}^{2+}$ behavior in either Me-DAPP ${ }^{2+}$ $\left(\lambda_{\max }=505 \mathrm{~nm}, \tau=17.6 \mathrm{~ns}, \Phi_{\mathrm{fl}}=66 \%\right.$, Figure S11) or Bn-DAPP $^{2+}\left(\lambda_{\max }=509 \mathrm{~nm}, \tau=19.8 \mathrm{~ns}, \Phi_{\mathrm{fl}}=60 \%\right.$, Figure S12), both of which show strong green fluorescence, so electron transfer from these species is unlikely (vide infra).

Some of the relevant photophysical data for all the compounds investigated are summarized in Table 1 , where the

Table 1. Photophysical Properties of the Reference Compounds and DAPPBox ${ }^{4+}$

\begin{tabular}{lcccc}
\multicolumn{1}{c}{ compound } & $\begin{array}{c}\lambda_{\text {abs }}(\mathrm{nm})^{a} / \\
\varepsilon_{\max }\left(10^{4} \mathrm{M}^{-1} \mathrm{~cm}^{-1}\right)\end{array}$ & $\lambda_{\text {ems }}(\mathrm{nm})^{a}$ & $\begin{array}{c}\tau(\mathrm{ns})^{b} / \\
\lambda_{\text {exc }}(\mathrm{nm})^{a}\end{array}$ & $\Phi_{\text {ems }}{ }^{c}$ \\
Me-ExBIPY $^{2+}$ & $323 / 3.15$ & 364 & $1.56 / 330$ & 0.69 \\
Me-DAPP $^{2+}$ & $438 / 5.66$ & 505 & $17.6 / 375$ & 0.66 \\
Bn-ExBIPY $^{2+}$ & $321 / 2.31$ & 378 & $0.062 / 330$ & 0.04 \\
Bn-DAPP $^{2+}$ & $443 / 5.74$ & 509 & $19.8 / 375$ & 0.60 \\
DAPPBox $^{4+}$ & $442 / 4.98$ & 520 & $19.5 / 374$ & 0.39
\end{tabular}

${ }^{a} \lambda_{\text {abs, }} \lambda_{\text {ems, }}$ and $\lambda_{\text {exc }}$ are the maximum absorption, emission, and excitation wavelengths. ${ }^{b} \tau$ is the fluorescence lifetime. ${ }^{c} \Phi_{\mathrm{ems}}$ is the fluorescence quantum yield.

emission maxima refer to the spectra corrected for instrument detection response. The UV/vis absorption spectrum of the model compounds Me-ExBIPY ${ }^{2+}$ and Me-DAPP ${ }^{2+}$, and the cyclophane DAPPBox ${ }^{4+}$, recorded in $\mathrm{MeCN}$ are shown in Figure 5. The absorption bands for Me-ExBIPY ${ }^{2+}$ occur at wavelengths shorter than $350 \mathrm{~nm}$, with the maximum centered on $323 \mathrm{~nm}$. The absorption spectrum for Me-DAPP ${ }^{2+}$ is characterized by an intense band in the visible region, which is assigned to the $S_{2} \leftarrow S_{0}$ electronic transition $\left(\lambda_{\max }=438 \mathrm{~nm}\right)$, and in the UV region $(<325 \mathrm{~nm})$, assigned to the $S_{\mathrm{n}} \leftarrow S_{0}$ electronic transition $\left(\lambda_{\max }=296 \mathrm{~nm}\right)$. The exact ordering of the upper state accessed in this transition is left unassigned. The absorption of the DAPPBox ${ }^{4+}$ exhibits minor differences when compared with the sum of the spectra of the corresponding model compounds, with only a slight red-shift of the absorption

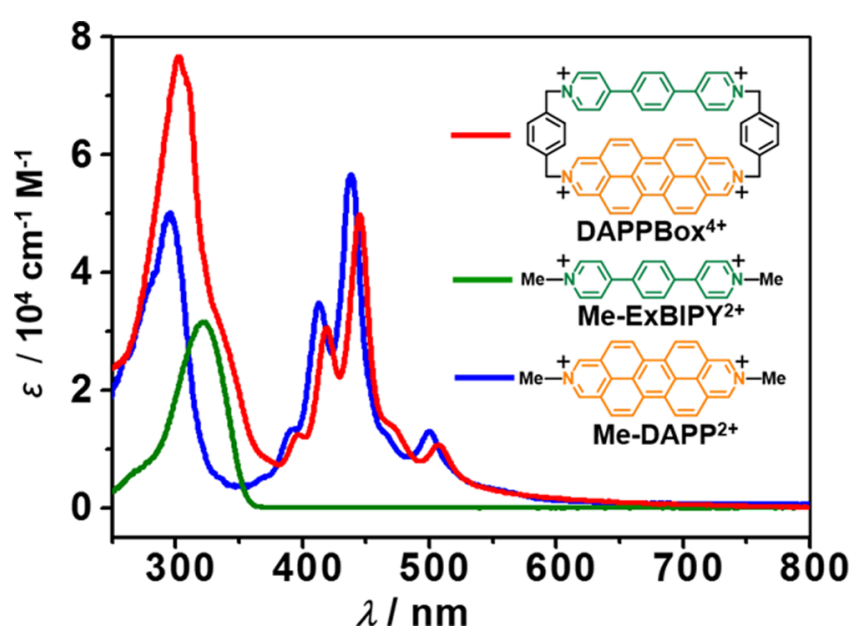

Figure 5. Absorption spectra of DAPPBox ${ }^{4+}, \mathrm{Me}-\mathrm{ExBIPY}^{2+}$, and Me-DAPP ${ }^{2+}$ in $\mathrm{MeCN}$ at room temperature. 
maximum $\left(\lambda_{\max }=446 \mathrm{~nm}\right)$. Importantly, from the reference compounds we see that at $330 \mathrm{~nm}$, approximately $86 \%$ of the net extinction coefficient of DAPPBox ${ }^{4+}$ can be assigned to the $\mathrm{ExBIPY}^{2+}$ subunit, while the remaining $14 \%$ can be attributed to the $\mathrm{DAPP}^{2+}$ absorption. Thus, we expect the excited states of each unit to participate in the dynamics following excitation.

Excited-State Dynamics. The excited-state dynamics of DAPPBox $^{4+}$ and its subunits were probed by femtosecond and nanosecond transient absorption (fsTA/nsTA) spectroscopies. The fsTA spectra (Figures S15a and 18a) of both Me-DAPP ${ }^{2+}$ and $\mathbf{B n}-\mathrm{DAPP}^{2+}$, following excitation at $330 \mathrm{~nm}$, show similar spectral features: the ground-state bleach appears at $505 \mathrm{~nm}$ overlapping with the singlet excited-state absorption band, which spans from 450 to $900 \mathrm{~nm}$ and at $1440 \mathrm{~nm}$. Me-DAPP ${ }^{2+}$ and $\mathbf{B n}-\mathbf{D A P P}{ }^{2+}$ both show rapid internal conversion to a hot $* S_{1}$ state, followed by structural relaxation prior to decay of their singlet states (Figures S15a and S18a). The 20 ns singletstate lifetimes for both $\mathrm{DAPP}^{2+}$ derivatives are longer than the maximum pump-probe delay time $(\sim 8 \mathrm{~ns})$ of the fsTA experiment. The corresponding nsTA spectra (Figures S15b and S18b) show that the ${ }^{1 *}$ Me-DAPP ${ }^{2+}$ and ${ }^{1} * \mathbf{B n}^{-D A P P}{ }^{2+}$ singlet states decay to their respective triplet states with time constants similar to those observed by time-resolved fluorescence; the triplet states then decay back to the ground state in approximately 250 and 220 ns, respectively, in air-equilibrated solutions at room temperature, presumably by collisional triplet-triplet annihilation and/or oxygen quenching. The reasonably intense triplet signals are commensurate with the moderate fluorescence quantum yields for $\mathbf{M e - D A P P}{ }^{2+}$ and $\mathbf{B n - D A P P}{ }^{2+}$, as well as with the previous observation ${ }^{10 c}$ of the triplet state of $\mathrm{DAPP}^{2+}$.

The transient absorption spectrum of Bn-ExBIPY ${ }^{2+}$ excited at $330 \mathrm{~nm}$ is shown in Figure S21. ${ }^{7 \mathrm{e}}$ The spectrum is initially dominated by an intense peak at $495 \mathrm{~nm}$ and a weaker feature around $1440 \mathrm{~nm}$, which are assigned to the $S_{\mathrm{n}} \leftarrow \mathrm{S}_{1}$ absorption. These features decay in $\tau=62 \pm 1 \mathrm{ps}$ as new bands peaking at 506 and $1110 \mathrm{~nm}$ appear. The spectra at this point strongly resemble ExBIPY ${ }^{+}, 7$, which confirms that fluorescence quenching of the ${ }^{1 *}$ Bn-ExBIPY ${ }^{2+}$ occurs by forward electron transfer. Back electron transfer occurs in $1200 \mathrm{ps,} \mathrm{with} \mathrm{some}$ fraction recombining to the triplet state $\left(\mathrm{T}_{\mathrm{n}} \leftarrow \mathrm{T}_{1}, 480 \mathrm{~nm}\right)$.

The fsTA spectra of DAPPBox ${ }^{4+}$ following $330 \mathrm{~nm}$ excitation appear (Figure 6a) very similar in both Me-DAPP ${ }^{2+}$ and Bn-DAPP $^{2+}$. The absorption band present at $\sim 1545 \mathrm{~nm}$ (Figure 6a) in the fsTA spectra of DAPPBox ${ }^{4+}$ persists for the entire singlet-state lifetime ( $\sim 20 \mathrm{~ns})$, which is most likely the $\mathrm{S}_{\mathrm{n}} \leftarrow \mathrm{S}_{1}$ excited-state absorption of the $\mathrm{DAPP}^{2+}$ unit. The most noticeable difference is a pronounced, rapid decay of the absorption at $495 \mathrm{~nm}$, with the appearance of a new absorption at $1150 \mathrm{~nm}$. The $495 \mathrm{~nm}$ signal decays on a slower time scale, which is similar to that observed for the singlet decay of excited $\mathrm{DAPP}^{2+}$ chromophores. The $1150 \mathrm{~nm}$ band appears within the $\sim 200 \mathrm{fs}$ instrument response and exhibits multiple decay components. As in the Me-DAPP ${ }^{2+}$ and $\mathbf{B n}-\mathbf{D A P P}{ }^{2+}$ reference compounds, ${ }^{1 * \mathrm{DAPP}^{2+}}$ decays to the triplet with an approximately $20 \mathrm{~ns}$ lifetime, and the triplet decays back to the ground state within $200 \mathrm{~ns}$. Notably, the kinetics are more complicated in DAPPBox ${ }^{4+}$ because of the overlapping groundstate absorptions at the excitation wavelength (Figure 6).

To remove complications from the parallel excitations at $330 \mathrm{~nm}$, we acquired the fsTA spectra of DAPPBox ${ }^{4+}$ following excitation at $505 \mathrm{~nm}$ (Figure 6b), and separately at $414 \mathrm{~nm}$ (Figure S19). The spectra resemble those of both Me-DAPP ${ }^{2+}$
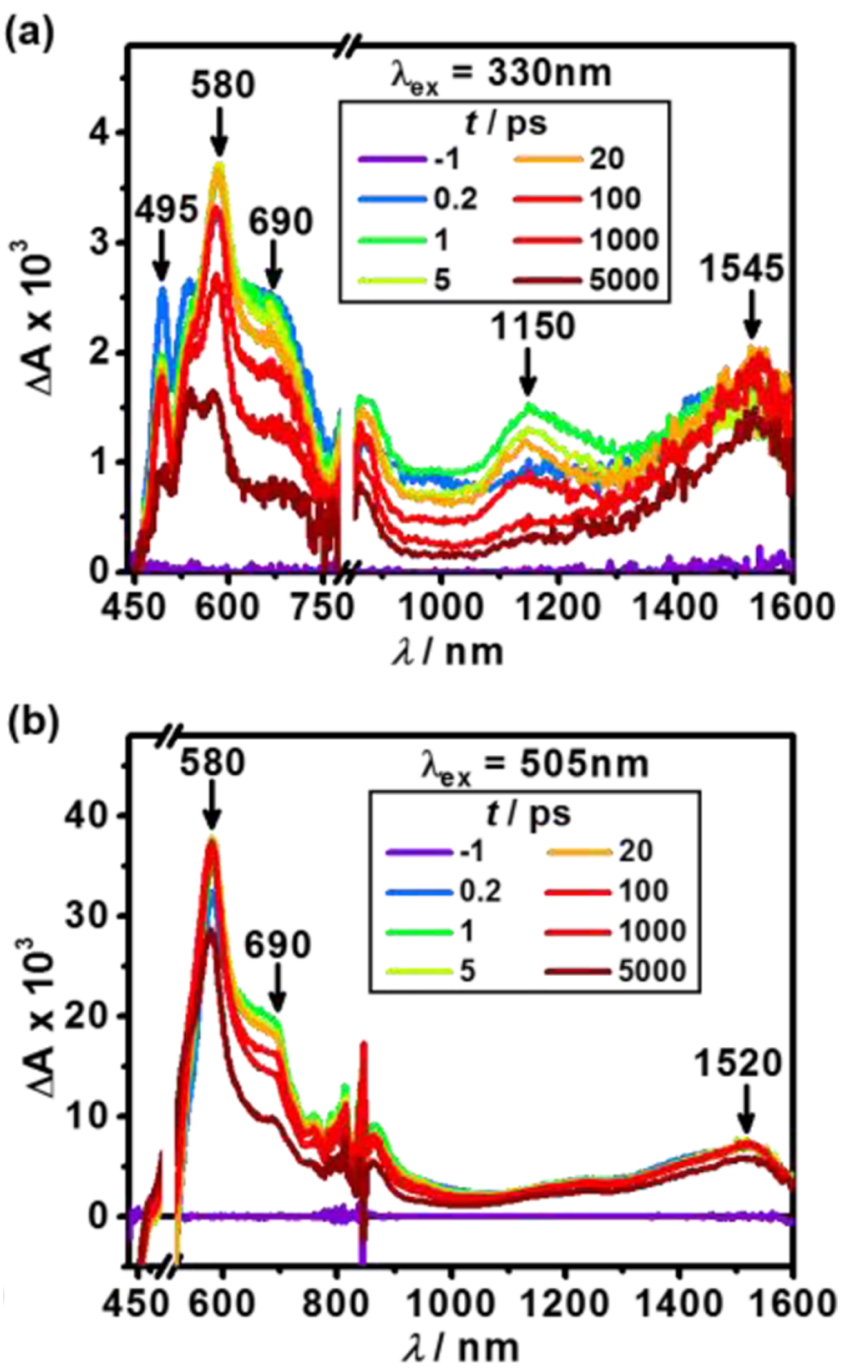

Figure 6. Visible and NIR spectra: (a) fsTA, $330 \mathrm{~nm}$ excitation, and (b) fsTA, $505 \mathrm{~nm}$ excitation spectra of DAPPBox ${ }^{4+}$ in $\mathrm{MeCN}$ at room temperature. In (a), the spectra beyond $850 \mathrm{~nm}$ are scaled by a factor of 2 for clarity.

and $\mathbf{B n}-\mathbf{D A P P}^{2+}$. Excitation at $414 \mathrm{~nm}$ again leads to rapid internal conversion to a hot $* S_{1}$ state. There is a slower structural relaxation due to the rigid conformation of the box, followed by similar singlet-state and triplet-state lifetimes, 18 and $250 \mathrm{~ns}$, respectively. Importantly, both the rapid decay of the peak at $495 \mathrm{~nm}$ and the new peak at $1150 \mathrm{~nm}$ that were observed when exciting at $330 \mathrm{~nm}$ are absent. This observation implies that exciting at lower photon energy shuts off some decay pathway(s), despite still exciting above the lowest energy excited state of DAPP ${ }^{2+}$.

Efficient Energy Transfer within DAPPBox ${ }^{4+}$. We expected an available EnT decay pathway (Figure 7a) from comparison of the emission spectra $\left(\lambda_{\text {exc }}=554 \mathrm{~nm}\right)$ in Figure $7 \mathrm{~b}$ of DAPPBox ${ }^{4+}$ with that of methylated reference compounds. Indeed, the emission spectrum of DAPPBox ${ }^{4+}$ is devoid of UV fluorescence and is dominated by green emission ( $\tau=19.5 \mathrm{~ns}$, $\Phi_{\mathrm{fl}}=39 \%$, Figures S9) at $517 \mathrm{~nm}$, even when the excitation occurs at $\mathrm{ExBIPY}^{2+}$ absorption wavelengths $\left(\lambda_{\mathrm{exc}}=339 \mathrm{~nm}\right)$. The quenching of ExBIPY ${ }^{2+}$ fluorescence at $380 \mathrm{~nm}$, accompanied by the enhancement of the $\mathrm{DAPP}^{2+}$ unit emission at $510 \mathrm{~nm}$ in the steady-state emission spectrum of DAPPBox ${ }^{4+}$, suggests efficient singlet EnT from the ExBIPY ${ }^{2+}$ to the 
(a)

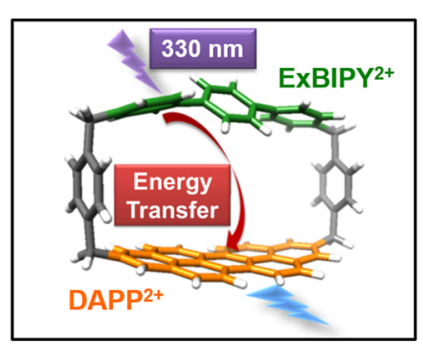

(c)

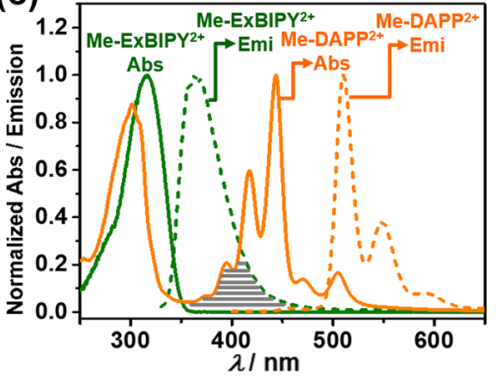

(b)

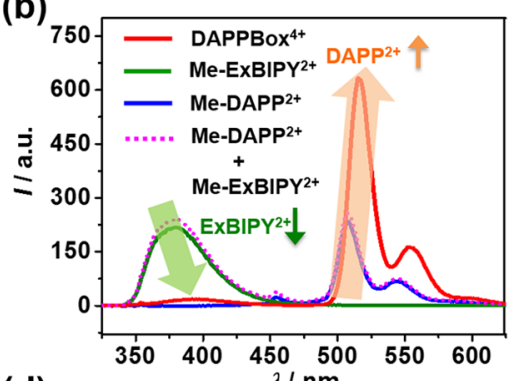

(d)

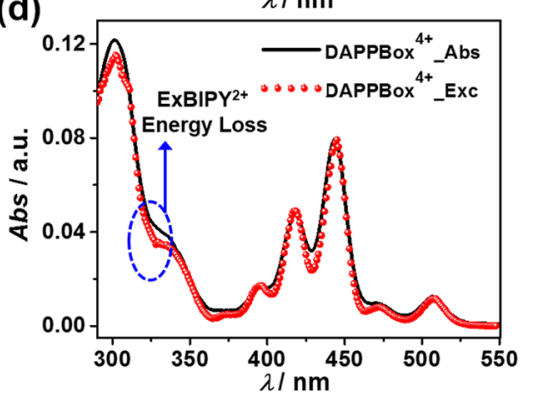

Figure 7. (a) Energy-transfer process within DAPPBox ${ }^{4+}$. (b) Emission spectra of DAPPBox ${ }^{4+}$, Me-ExBIPY $^{2+}$, Me-DAPP $^{2+}$, and a physical mixture of Me-ExBIPY ${ }^{2+}$ and Me-DAPP ${ }^{2+}$ in MeCN upon excitation at $339 \mathrm{~nm}, 1.6 \mu \mathrm{M}$. (c) Normalized spectra showing the overlap between emission of the Me-ExBIPY ${ }^{2+}$ and the absorption of the Me-DAPP ${ }^{2+}$. (d) Overlap between the excitation spectrum $\left(\lambda_{\text {em }}=554 \mathrm{~nm}\right)$ and the UV/vis absorption spectrum of DAPPBox ${ }^{4+}$.

(a)

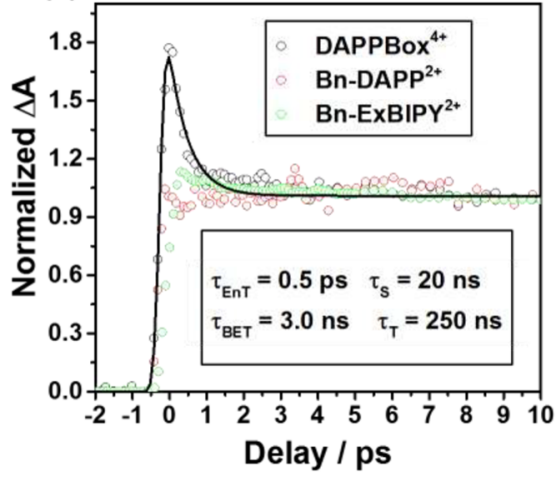

(b)

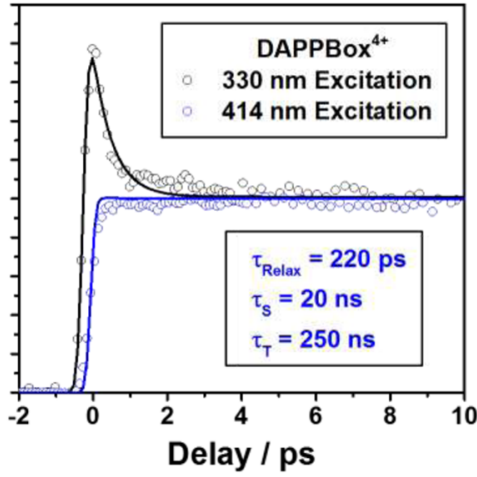

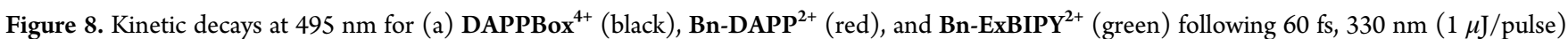
excitation and (b) DAPPBox ${ }^{4+}$ excited at $330 \mathrm{~nm}$ (black) and $414 \mathrm{~nm}$ (blue). Only the first $10 \mathrm{ps}$ are shown in order to highlight the ultrafast decay of the DAPPBox ${ }^{4+}$ trace, which is not present in the reference compounds. The fit of the full DAPPBox ${ }^{4+}$ kinetic decay is also shown; nanosecond lifetimes obtained from nsTA spectroscopy (Figures S35-S37). All traces were normalized to their respective values at 8 ps.

$\mathrm{DAPP}^{2+}$ unit within the cyclophane. Such relative quenching and enhancement were not observed in an equimolar physical mixture of Me-ExBIPY ${ }^{2+}$ and Me-DAPP ${ }^{2+}$, indicating that efficient EnT processes occur only within the cyclophane. This result can be rationalized by consideration of the conformation of the cyclophane which imparts (i) a reasonable spatial separation (7.1 Å, measured from single crystal structure, Figure 2b) between the energy donor $\left(\mathrm{ExBIPY}^{2+}\right)$ and acceptor $\left(\mathrm{DAPP}^{2+}\right)$ in DAPPBox $^{4+}$, (ii) a face-to-face chromophore arrangement that aligns the transition dipole moments, and (iii) rigidity that limits conformational flexibility. The high efficiency of EnT is also in line with the favorable overlap (Figure $7 \mathrm{c}$ ) of the emission spectra of Me-ExBIPY ${ }^{2+}$ and the absorption spectra of Me-DAPP ${ }^{2+}$, as required for Förster energy transfer. Moreover, the almost perfect matching of the fluorescence excitation spectrum $\left(\lambda_{\text {em }}=554 \mathrm{~nm}\right)$ and the UV/vis absorption spectrum (Figure $7 \mathrm{~d}$ ) of DAPPBox ${ }^{4+}$, once again, corroborates the assignment of EnT process within DAPPBox ${ }^{4+}$.
The fsTA spectra support the observation of efficient energy transfer from ${ }^{1} * \mathrm{ExBIPY}^{2+}$ to $\mathrm{DAPP}^{2+}$ deduced from the fluorescence data. The fsTA spectra of DAPPBox ${ }^{4+}$ following $330 \mathrm{~nm}$ excitation show (Figure 6a) increased intensity of the absorption band at $495 \mathrm{~nm}$ during the first few picoseconds after excitation compared to those of Bn-DAPP ${ }^{2+}$ (Figure S18a), and DAPPBox $^{4+}$ following $414 \mathrm{~nm}$ excitation (Figure S19a), suggesting the brief appearance of the locally excited state ${ }^{1}$ ExBIPY $^{2+}$ (Figure S21a). This relative enhancement does

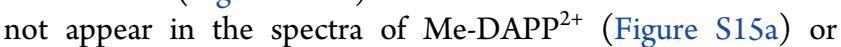
Bn-DAPP $^{2+}$ (Figure S18a) nor does it appear in the spectra of DAPPBox $^{4+}$ when only the DAPP ${ }^{2+}$ unit is excited (Figures $6 \mathrm{~b}$ and S19a). Figure 8a shows a comparison of the kinetics at $495 \mathrm{~nm}$ for DAPPBox ${ }^{4+}$ with Me-ExBIPY ${ }^{2+}$ and Me-DAPP ${ }^{2+}$ under identical conditions, while Figure $8 \mathrm{~b}$ shows the kinetic fits for DAPPBox ${ }^{4+}$ when the ExBIPY ${ }^{2+}$ and $\mathrm{DAPP}^{2+}$ units are excited at 330 and $414 \mathrm{~nm}$, respectively. Scatter of the $505 \mathrm{~nm}$ pump prevented the use of that data set for this comparison. 
Upon fitting the kinetic decay at $495 \mathrm{~nm}$, we determined that the energy transfer occurs with a 0.5 ps time constant, which shows that energy transfer from ${ }^{1}{ }^{*} \mathrm{ExBIPY}^{2+}$ to $\mathrm{DAPP}^{2+}$ is highly efficient and outcompetes other deactivation pathways previously observed ${ }^{9}$ in ExBox $^{4+}$.

The rate of resonance EnT can be calculated using Förster theory based on the spectral overlap between the absorption of the Me-ExBIPY ${ }^{2+}$ acceptor and the emission of the Me-DAPP ${ }^{2+}$ donor. We have calculated the Förster EnT rate with the PhotochemCAD software package, ${ }^{19}$ using the steadystate spectra, the fluorescence quantum yield of the donor, the distance between the donor and acceptor from the XRD data, ${ }^{13}$ and the refractive index of the $\mathrm{MeCN}$ as inputs. The fixed parallel orientation of the donor and acceptor units in DAPPBox $^{4+}$ leads to an orientation factor of $\kappa^{2}=1$, as opposed to the typical value of $\kappa^{2}=2 / 3$ for freely rotating molecules where reorientation occurs faster than the emission time scale. The calculated EnT rate is therefore $k_{\mathrm{EnT}}=(0.43 \mathrm{ps})^{-1}$, a value which is in excellent agreement with the experimental result of $k_{\mathrm{EnT}}=(0.5 \mathrm{ps})^{-1}$ and supports the Förster mechanism. Dexter energy transfer cannot be entirely ruled out, however, since there may be sufficient electronic wave function overlap between the donor and acceptor units to enable ultrafast electron transfer (vide infra).

Computational modeling of ${ }^{1 * D A P P B o x}{ }^{4+}$ using timedependent density functional theory (TDDFT) predicts the first two major absorption peaks at 451 and $352 \mathrm{~nm}$, which correspond to ${ }^{1 *} \mathrm{DAPP}^{2+}$ and ${ }^{1 *} \mathrm{ExBIPY}^{2+}$, respectively, in good agreement with experimental observations. The electronic configuration of ${ }^{1 *} \mathrm{ExBIPY}^{2+}$ is mainly composed of contributions from molecular orbitals (MOs) of the ExBIPY ${ }^{2+}$ unit, while the $451 \mathrm{~nm}$ excitation of the $\mathrm{DAPP}^{2+}$ unit involves (Figure S41) contributions from the MOs of the $p$-xylylene linkers and the $\mathrm{DAPP}^{2+}$ unit. The total quantum yield from emission of the DAPPBox ${ }^{4+}$ can be calculated through the product of (i) the EnT efficiency of DAPPBox ${ }^{4+}$ (98\%, based on competing rates of the ExBIPY ${ }^{2+}$ excited-state lifetime of $1.56 \mathrm{~ns}$, and the Bn-ExBIPY ${ }^{2+}$ charge-transfer time of $60 \mathrm{ps}$ ), (ii) the photon absorption efficiency of $\mathrm{ExBIPY}^{2+}$ at $330 \mathrm{~nm}$ (84\%, based on extinction coefficients), and (iii) the fluorescence quantum yield of $\mathbf{B n}^{-D A P P}{ }^{2+}(0.66$, excited at $375 \mathrm{~nm})$. The calculated quantum yield of $0.54(=0.98 \times$ $0.84 \times 0.66)$, for the cyclophane is slightly higher than the corresponding measured fluorescence quantum yield of 0.39 . The difference between the calculated and experimental results can be explained by the difference between the quantum yields of the units in the cyclophane and those of the ExBIPY ${ }^{2+}$ and $\mathrm{DAPP}^{2+}$ reference compounds. Other possible means whereby the quantum yield of the cyclophane is decreased may arise from alternative decay pathways, e.g., an increased rate of internal conversion. We, however, have no direct evidence for any other competitive process.

Ultrafast Electron Transfer within DAPPBox ${ }^{4+}$. The overlapping $\mathrm{DAPP}^{2+}$ unit absorption at $330 \mathrm{~nm}$ leads to parallel excited-state populations of ${ }^{1 *} \mathrm{ExBIPY}^{2+}$ and ${ }^{1 *} \mathrm{DAPP}^{2+}$ within DAPPBox ${ }^{4+}$, with the majority of the excitation on the ExBIPY moiety. With the observation of ultrafast energy transfer, we expect no significant yield of charge transfer from the $p$-xylylene linker to ${ }^{1 *} \mathrm{ExBIPY}^{2+}$ in the DAPPBox ${ }^{4+}$ cyclophane, since this electron transfer occurs with a 60 ps time constant in Bn-ExBIPY ${ }^{2+}$ and 240 ps in the ExBox ${ }^{4+}$ cyclophane. We also do not observe any of the characteristic absorption bands ${ }^{10 c}$ for $\mathrm{DAPP}^{+}$, indicating that ${ }^{1 *} \mathrm{ExBIPY}^{2+}$ does not reduce the
Scheme 2. Schematic Representation of the Photodriven Charge Redistribution Yielding the DAPP $^{3+\bullet}-$ ExBIPY $^{+}$ Radical Ion-Pair in DAPPBox ${ }^{4+}$

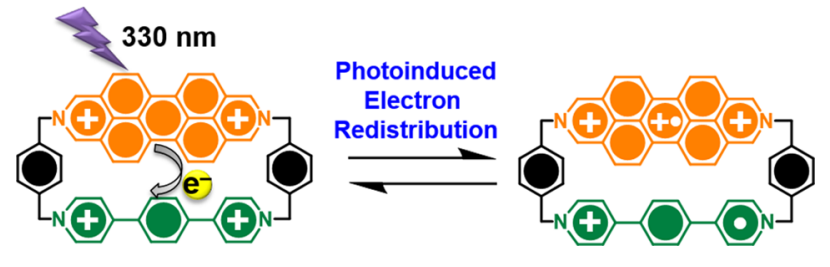

$\mathrm{DAPP}^{2+}$ moiety within DAPPBox ${ }^{4+}$. If either of these chargetransfer pathways were significant, the fluorescence quantum yield and lifetime of the cyclophane would be substantially lower. ${ }^{10 \mathrm{c}}$

However, the appearance of the $1150 \mathrm{~nm}$ band that is similar to the characteristic ${ }^{14,6 c, 9}$ of ExBIPY ${ }^{+\bullet}$ absorption alongside the DAPP suggests that the smaller of these populations decays by ultrafast electron transfer. The red-shift of that band may arise due to Stark effects of the $\mathrm{DAPP}^{2+}$ moiety in close proximity. The $1150 \mathrm{~nm}$ band appears immediately following excitation and rises over the next few picoseconds. The fit of the DAPPBox $^{4+}$ spectra (Figure S37) indicates that the forward electron transfer from $1^{*} \mathrm{DAPP}^{2+}$ to $\mathrm{ExBIPY}^{2+}$ occurs in $\tau_{\mathrm{FET}}=$ $1.5 \mathrm{ps}$. Therefore, we rule out transfer from the $p$-xylyl linker to ExBIPY (vide supra). Back electron transfer then follows in $\tau_{\mathrm{BET}}=3.2 \pm 0.8 \mathrm{~ns}$. The back-electron-transfer time from the global fits of the fsTA data is consistent with the $\tau=2.2 \pm$ $1.2 \mathrm{~ns}$ decay observed in the nsTA data (Figure S40). Additionally, as discussed above, no evidence for electron transfer from the $p$-xylyl linker to DAPP ${ }^{2+}$ was observed in the $\mathrm{Bn}-\mathrm{DAPP}^{2+}$ reference compound, suggesting that the $1150 \mathrm{~nm}$ absorption originates from the $\mathrm{DAPP}^{3+\bullet}-\mathrm{ExBIPY}^{+\bullet}$ radical ion pair (Scheme 2).

It should be noted that there is an absorption band present at $1520 \mathrm{~nm}$. However, this band is not an indicator of electron transfer but rather is characteristic of the $S_{1}$ state for Bn-DAPP $^{2+}$ (Figure S19) as a result of the $505 \mathrm{~nm}$ excitation, since (i) this $1520 \mathrm{~nm}$ absorption band in the NIR persists for the entire singlet lifetime ( $\sim 20 \mathrm{~ns}$ as confirmed by time-resolved fluorescence) in the case of both Bn-DAPP ${ }^{2+}$ (Figure S19) and DAPPBox $^{4+}$ (Figure 6b) when excited at $505 \mathrm{~nm}$, and (ii) this band is also similar to the $1430 \mathrm{~nm}$ absorption band for Me-DAPP $^{2+}$ (no electron transfer possible when excited at $505 \mathrm{~nm})$, shifted, however, by $\sim 50 \mathrm{meV}\left(\sim 400 \mathrm{~cm}^{-1}\right)$. The red shift of the band wavelength can be explained by substituent effects.

The excitation wavelength dependence of the fsTA signal indicates that electron transfer originates from a higher excited state of $\mathrm{DAPP}^{2+}$. The cyclic voltammogram in Figure S7 shows that the oxidation potential of $\mathrm{DAPP}^{2+}$ in $\mathrm{MeCN}$ is $1.69 \mathrm{~V}$ vs $\mathrm{Ag} / \mathrm{AgCl}$, while the reduction potential of $\mathrm{ExBIPY}^{2+}$ has been reported previously as $-0.65 \mathrm{~V}$ vs $\mathrm{Ag} / \mathrm{AgCl}{ }^{8 \mathrm{a}}$ Using the Weller equation, we can estimate the energy of the $\mathrm{DAPP}^{3+\bullet}-\mathrm{ExBIPY}^{+}$ radical ion-pair state in $\mathrm{MeCN}$ as $\Delta G_{\mathrm{IP}} \approx E_{\mathrm{ox}}-E_{\text {red }}=2.34 \mathrm{eV}$, and the free energy of photoinduced electron transfer from the various accessible excited states with singlet energy $E_{\mathrm{S}}$ is $\Delta G_{\mathrm{ET}} \approx \Delta G_{\mathrm{IP}}-E_{\mathrm{S}}$. From this estimate, we see that electron transfer from $S_{1}$ of $\mathrm{DAPP}^{2+}$ at $2.46 \mathrm{eV}$ is only slightly thermodynamically favorable. However, the solvent reorganization energy is around $\sim 1 \mathrm{eV}$ in this solvent, ${ }^{6 \mathrm{f}}$ and Marcus theory predicts that the ET rate should be relatively slow. It is worth noting that the ion-pair energy obtained from the Weller 


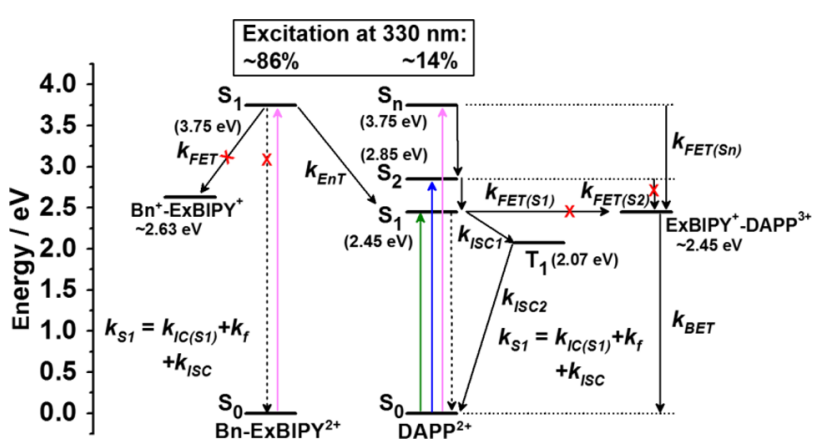

Figure 9. Schematic of the excited-state relaxation pathways in DAPPBox $^{4+}$ at different excitation wavelengths (IC $=$ internal conversion; ISC = intersystem crossing; $\mathrm{f}=$ fluorescence; $\mathrm{FET}=$ forward electron transfer; BET = back electron transfer; EnT = energy transfer). Dashed arrows indicated radiative transitions. Red X's indicate kinetically unfavorable processes.

equation is likely an overly simplistic approximation. From DFT (see Supporting Information for details), we calculate the lowest energy ion-pair state to be around $2.47 \mathrm{eV}$; the $\sim+0.13$ $\mathrm{eV}$ difference from the Weller estimate is enough to make ET from the $\mathrm{DAPP}^{2+} \mathrm{S}_{1}$ state thermodynamically unfavorable.

Interestingly, no ET is observed when exciting to the next higher energy excited state at $505 \mathrm{~nm}\left(S_{1} \leftarrow S_{0}\right.$ transition), or even $414 \mathrm{~nm}$ (higher vibrionic band of the $\sim 2.85 \mathrm{eV} \mathrm{S} \mathrm{S}_{2} \leftarrow \mathrm{S}_{0}$ transition), even though $\Delta G_{\mathrm{ET}}$ is more negative. It is likely that the rate of internal conversion between the $S_{2}$ and $S_{1}$ states $\left(\Delta E_{\mathrm{S}}=-0.40 \mathrm{eV}\right)$ is substantially faster than that of ET, effectively shutting off the latter process. Thus, we rule out ET from the lower energy excited states of $\mathrm{DAPP}^{2+}$ as a major source of the ion-pair population.

Only when exciting at $330 \mathrm{~nm}(3.76 \mathrm{eV})$ do we observe the radical ion-pair absorption, implying that ET is at least competitive with other decay pathways and is perhaps the dominant process from this state. From the energy gap law for nonradiative decay processes, we expect internal conversion from this state $\left(S_{n}\right)$ to the lower lying $S_{2}$ state (with gap $\Delta E_{S}=$ $-0.91 \mathrm{eV})$ to be slower than that from $S_{2}$ to $S_{1}$. At the same time, Marcus theory predicts that with $\Delta G_{\mathrm{ET}} \approx-1.41 \mathrm{eV}$ $(-1.38 \mathrm{eV}$ using the DFT ion-pair energy), the ET rate from the vertically prepared $S_{n}$ state will be much closer to the top of the Marcus rate vs free energy curve, and faster than that for ET from $S_{2}$ or $S_{1}$. Thus, the combination of faster ET and slower IC processes leads to measurable population of the ion-pair state when exciting at $330 \mathrm{~nm}$; the slower internal conversion from $S_{n}$ serves as a minor "bottleneck" for relaxation, allowing ET to proceed. The excited-state dynamics of DAPPBox ${ }^{4+}$ at each excitation wavelength are summarized in Figure 9.

\section{CONCLUSION}

We report the stepwise synthesis of a rationally designed multichromophoric donor-acceptor cyclophane, DAPPBox ${ }^{4+}$. We demonstrate that the use of pyrene as a template favors the formation of this constitutionally asymmetric cyclophane in $35 \%$ yield. The structure of DAPPBox ${ }^{4+}$ was determined by ${ }^{1} \mathrm{H}$ NMR spectroscopy, high-resolution mass spectrometry in solution, and single-crystal X-ray diffraction analysis in the solid state. Investigations carried out on the photophysical properties of the cyclophane excited at $330 \mathrm{~nm}$ by means of steady-state as well as time-resolved absorption and emission spectroscopies reveal not only efficient energy transfer from the ${ }^{1 *} \mathrm{ExBIPY}^{2+}$ to the $\mathrm{DAPP}^{2+}$ unit in $\tau=0.5 \mathrm{ps}$, but also the existence of an efficient, ultrafast intramolecular electron-transfer pathway: ${ }^{*} \mathrm{DAPP}^{2+}-\mathrm{ExBIPY}^{2+} \rightarrow \mathrm{DAPP}^{3+}-\mathrm{ExBIPY}^{+\bullet}$ radical ion pair in $\tau=1.5$ ps. The photophysical investigations were also supported by TDDFT results. This class of constitutionally asymmetric cyclophanes may have considerable potential for integration into solar energy conversion and organic electronics. In the context of artificial photosynthesis, the next logical step toward utilizing DAPPBox ${ }^{4+}$ will be to bind different guest molecules inside the cyclophane, forming 1:1 complexes, which can serve to modulate the electrontransfer rates.

\section{EXPERIMENTAL SECTION}

3. 3,4,9,10-Tetrakis(chloromethyl)perylene ${ }^{15}$ (2) $(623 \mathrm{mg}, 4.53 \mathrm{mmol})$ was added with stirring to a solution of 4-(aminomethyl)benzenemethanol $^{16}$ (1) (135 mg, $\left.0.302 \mathrm{mmol}\right)$ in $\mathrm{Me}_{2} \mathrm{SO}(8 \mathrm{~mL})$ at room temperature. After $5 \mathrm{~h}$, deionized $\mathrm{H}_{2} \mathrm{O}(100 \mathrm{~mL})$ was added to the reaction mixture, forming a dark yellow precipitate, which was filtered off and then washed with deionized $\mathrm{H}_{2} \mathrm{O}$ and a small amount of EtOH, before being dried under vacuum to afford $(147 \mathrm{mg}, 85 \%)$ a dark yellow solid 3. The product was used without further purification in the next step. ${ }^{1} \mathrm{H}$ NMR $\left(500 \mathrm{MHz}, \mathrm{CD}_{3} \mathrm{SOCD}_{3}\right): \delta_{\mathrm{H}}=8.20(\mathrm{~d}, J=$ $7.7 \mathrm{~Hz}, 4 \mathrm{H}), 7.32(\mathrm{q}, J=8.0 \mathrm{~Hz}, 8 \mathrm{H}), 7.24(\mathrm{~d}, J=7.7 \mathrm{~Hz}, 4 \mathrm{H}), 4.50$ $(\mathrm{d}, J=5.7 \mathrm{~Hz}, 4 \mathrm{H}), 3.86(\mathrm{~s}, 8 \mathrm{H}), 3.75(\mathrm{~s}, 4 \mathrm{H}) .{ }^{13} \mathrm{C}$ NMR $(126 \mathrm{MHz}$, $\left.\mathrm{CD}_{3} \mathrm{SOCD}_{3}\right): \delta_{\mathrm{C}}=140.8,135.7,132.7,128.1,125.1,122.6,119.1$, $62.1,60.3,55.2$. More detailed discussion of this reaction is available in the Supporting Information.

4.2PF 6 . DDQ ( $240 \mathrm{mg}, 1.057 \mathrm{mmol})$ was added to a dark yellow solution of $3(100 \mathrm{mg}, 0.182 \mathrm{mmol})$ in DMF $(8 \mathrm{~mL})$ at room temperature. The color of the reaction mixture became dark brown upon the addition of the DDQ. After stirring for $8 \mathrm{~h}$, a satd aqueous solution $(100 \mathrm{~mL})$ of $\mathrm{NH}_{4} \mathrm{PF}_{6}$ was added to the reaction mixture. The resulting precipitate was filtered off and then washed with deionized $\mathrm{H}_{2} \mathrm{O}$ and a small amount of EtOH, before being dried under vacuum to afford $(138 \mathrm{mg}, 90 \%)$ a dark brown solid $4 \cdot 2 \mathrm{PF}_{6}$. The crude product was dissolved in $\mathrm{MeCN}$ in order to exchange the anions from $\mathrm{PF}_{6}{ }^{-}$to $\mathrm{Cl}^{-}$by addition of an excess of tetrabutylammonium chloride (TBACl). The crude dibromide was then subjected to reverse-phase HPLC, starting with $\mathrm{H}_{2} \mathrm{O} / 0.1 \%$ TFA as eluent and adding up to $100 \%$ of $\mathrm{MeCN} / 0.1 \% \mathrm{TFA}$ to the eluent within $40 \mathrm{~min}$. The pure fractions were collected and concentrated under vacuum. The residue was dissolved in $\mathrm{H}_{2} \mathrm{O}$, followed by the addition of $\mathrm{NH}_{4} \mathrm{PF}_{6}$ to yield pure 4 . $2 \mathrm{PF}_{6}(124 \mathrm{mg}, 80 \%) .{ }^{1} \mathrm{H}$ NMR $\left(500 \mathrm{MHz}, \mathrm{CD}_{3} \mathrm{SOCD}_{3}\right): \delta_{\mathrm{H}}=10.39$ (s, 4H), $9.94(\mathrm{~s}, 4 \mathrm{H}), 8.95(\mathrm{~s}, 4 \mathrm{H}), 7.72(\mathrm{~d}, J=7.9 \mathrm{~Hz}, 4 \mathrm{H}), 7.45$ (d, $J=7.8 \mathrm{~Hz}, 4 \mathrm{H}), 6.41(\mathrm{~s}, 4 \mathrm{H}), 4.53(\mathrm{~s}, 4 \mathrm{H}) .{ }^{13} \mathrm{C}$ NMR $(126 \mathrm{MHz}$, $\left.\mathrm{CD}_{3} \mathrm{SOCD}_{3}\right): \delta_{\mathrm{C}}=150.4,138.4,128.3,128.3,126.5,126.2,113.7$, $100.3,61.8$.

BrDAPP. $2 \mathrm{PF}_{6} \cdot 4 \cdot 2 \mathrm{PF}_{6}(100 \mathrm{mg}, 0.116 \mathrm{mmol})$ was dissolved in $\mathrm{MeCN}(15 \mathrm{~mL})$, and $\mathrm{PBr}_{3}(100 \mu \mathrm{L}, 1.063 \mathrm{mmol})$ was added slowly while stirring at room temperature overnight. A saturated aqueous solution $(100 \mathrm{~mL})$ of $\mathrm{NH}_{4} \mathrm{PF}_{6}$ was added to quench the reaction, resulting in the formation of a reddish brown solid. The resulting precipitate was filtered off and then washed with deionized $\mathrm{H}_{2} \mathrm{O}$ and a small amount of $\mathrm{EtOH}$, before being dried under vacuum to afford $(80 \mathrm{mg}, 70 \%)$ a reddish brown solid BrDAPP.2PF $6 .{ }^{1} \mathrm{H}$ NMR $\left(500 \mathrm{MHz}, \mathrm{CD}_{3} \mathrm{SOCD}_{3}\right): \delta_{\mathrm{H}}=10.44(\mathrm{~s}, 4 \mathrm{H}), 10.04(\mathrm{~d}, J=9.6 \mathrm{~Hz}$, $4 \mathrm{H}), 9.04(\mathrm{~d}, J=9.3 \mathrm{~Hz}, 4 \mathrm{H}), 7.72(\mathrm{~d}, J=7.9 \mathrm{~Hz}, 4 \mathrm{H}), 7.60(\mathrm{~d}, J=$ $8.0 \mathrm{~Hz}, 4 \mathrm{H}), 6.45(\mathrm{~s}, 4 \mathrm{H}), 4.74(\mathrm{~s}, 4 \mathrm{H}) .{ }^{13} \mathrm{C}$ NMR $(126 \mathrm{MHz}$, $\left.\mathrm{CD}_{3} \mathrm{SOCD}_{3}\right): \delta_{\mathrm{C}}=161.7,138.8,129.5,128.7,127.7,127.2,126.1$, 63.7, 39.0, 33.0

DAPPBox $4 \mathrm{PF}_{6}$. A solution of BrDAPP. $2 \mathrm{PF}_{6}$ (1 equiv), ExBIPY ( 1 equiv), and pyrene (6 equiv) in dry $\mathrm{MeCN}$ was stirred at room temperature for 7 days. Excess of TBACl was added to quench the reaction, and the resulting crude precipitate was then dissolved in $\mathrm{H}_{2} \mathrm{O}$. The pyrene template was removed by continuous liquid-liquid extraction with $\mathrm{CHCl}_{3}$ and $\mathrm{H}_{2} \mathrm{O}$ over 3 days. The aqueous phase was concentrated to a small volume and then subjected to reverse-phase 
HPLC, starting with $\mathrm{H}_{2} \mathrm{O} / 0.1 \%$ TFA as eluent, and adding up to $100 \%$ of $\mathrm{MeCN} / 0.1 \% \mathrm{TFA}$ as eluent within $45 \mathrm{~min}$. The pure fractions were collected and concentrated in vacuum. The residue was dissolved in $\mathrm{H}_{2} \mathrm{O}$, followed by the addition of $\mathrm{NH}_{4} \mathrm{PF}_{6}$ to afford pure DAPPBox$4 \mathrm{PF}_{6}$ in $35 \%$ yield. ${ }^{1} \mathrm{H}$ NMR $\left(500 \mathrm{MHz}, \mathrm{CD}_{3} \mathrm{CN}\right): \delta_{\mathrm{H}}=9.96(\mathrm{~s}, 4 \mathrm{H})$, $9.65(\mathrm{~s}, 4 \mathrm{H}), 8.86(\mathrm{~s}, 4 \mathrm{H}), 8.65(\mathrm{~d}, J=6.1 \mathrm{~Hz}, 4 \mathrm{H}), 7.95-7.83$ $(\mathrm{m}, 8 \mathrm{H}), 7.61(\mathrm{~d}, J=8.0 \mathrm{~Hz}, 4 \mathrm{H}), 7.54(\mathrm{~s}, 4 \mathrm{H}), 6.28(\mathrm{~s}, 4 \mathrm{H}), 5.62$ $(\mathrm{s}, 4 \mathrm{H}) \cdot{ }^{13} \mathrm{C}$ NMR $\left(126 \mathrm{MHz}, \mathrm{CD}_{3} \mathrm{CN}\right): \delta_{\mathrm{C}}=144.5,138.2,130.8$, 130.6, 129.3, 127.0, 126.0, 66.4, 64.5. HRMS (ESI) for DAPPBox$4 \mathrm{PF}_{6}$; Calcd for $\mathrm{C}_{56} \mathrm{H}_{40} \mathrm{~F}_{24} \mathrm{~N}_{4} \mathrm{P}_{4}: m / z=1203.2174\left[\mathrm{M}-\mathrm{PF}_{6}\right]^{+}$; Found: $1203.2173\left[\mathrm{M}-\mathrm{PF}_{6}\right]^{+}$; $529.1266\left[\mathrm{M}-2 \mathrm{PF}_{6}\right]^{2+}$; Found: $529.1263\left[\mathrm{M}-2 \mathrm{PF}_{6}\right]^{2+} ; 304.4293\left[\mathrm{M}-3 \mathrm{PF}_{6}\right]^{3+}$; Found: 304.4293 $\left[\mathrm{M}-3 \mathrm{PF}_{6}\right]^{3+}$.

Single-Crystal X-ray Diffraction (XRD). DAPPBox.4TFA. (a) Method. A $0.25 \mathrm{mM}$ MeCN solution of DAPPBox-4TFA was filtered through a $0.45-\mu \mathrm{m}$ syringe filter into VWR culture tubes. The culture tubes were placed in one closed $20 \mathrm{~mL}$ scintillation vial containing $3 \mathrm{~mL} i \mathrm{Pr}_{2} \mathrm{O}$. After 7 days, reddish brown crystals of DAPPBox-4TFA were obtained. A suitable crystal was selected and mounted in inert oil and transferred to the cold gas stream of a Bruker Kappa APEX CCD area detector diffractometer. The crystal was kept at $100 \mathrm{~K}$ during data collection. Using Olex2, ${ }^{17}$ the structure was solved with the $\mathrm{XM}^{18}$ structure solution program using Dual Space and refined with the XL refinement package using least-squares minimization.

(b) Crystal Parameters. DAPPBox-4TFA, orange needles, $\mathrm{M}=$ 2442.00 , crystal size $0.124 \times 0.122 \times 0.02 \mathrm{~mm}^{3}$, triclinic, space group $P \overline{1} ; a=13.3141(7), b=21.1146(10)$, and $c=23.4964(13) \AA ; \alpha=$ 96.654(4), $\beta=99.661(4)$, and $\gamma=105.932(4)^{\circ} ; V=6169.5(6) \AA^{3}, \rho_{\text {calc }}$ $=1.315, T=100 \mathrm{~K}, Z=2 ; R_{1}\left(F^{2}>2 \sigma F^{2}\right)=0.0982, \mathrm{w} R_{2}=0.2196$. Out of 26407 reflections, a total of 25028 were unique. Crystallographic data (excluding structure factors) for this structure have been deposited with the Cambridge Crystallographic Data Center as supplementary publication no. CCDC 1480961.

\section{ASSOCIATED CONTENT}

\section{S Supporting Information}

The Supporting Information is available free of charge on the ACS Publications website at DOI: 10.1021/jacs.6b13223.

Experimental details, including synthesis, NMR, UV/vis/ NIR, fluorescence emission and excitation, fsTA data, and electrochemical experiments (PDF)

$\mathrm{X}$-ray crystallographic data for pyrene $C \mathrm{DAPPBox} \cdot 4 \mathrm{PF}_{6}$ (CIF)

\section{AUTHOR INFORMATION}

\section{Corresponding Authors}

*m-wasielewski@northwestern.edu

*stoddart@northwestern.edu

\section{ORCID}

Ryan M. Young: 0000-0002-5108-0261

Hai Xiao: 0000-0001-9399-1584

Peng Li: 0000-0002-4273-4577

Jiawang Zhou: 0000-0002-2399-0030

Tao Cheng: 0000-0003-4830-177X

William A. Goddard III: 0000-0003-0097-5716

Omar K. Farha: 0000-0002-9904-9845

Joseph T. Hupp: 0000-0003-3982-9812

Michael R. Wasielewski: 0000-0003-2920-5440

J. Fraser Stoddart: 0000-0003-3161-3697

\section{Author Contributions}

"X.G. and R.M.Y. contributed equally.

\section{Notes}

The authors declare no competing financial interest.

\section{ACKNOWLEDGMENTS}

This research was conducted as part of the Joint Center of Excellence in Integrated Nanosystems at King Abdulaziz City for Science and Technology (KACST) and Northwestern University (NU). The authors thank both KACST and NU for their continued support of this research. This work was also supported by the Chemical Sciences, Geosciences, and Biosciences Division, Office of Basic Energy Sciences, U.S. Department of Energy (DOE) under grant no. DE-FG0299ER14999 (M.R.W.). J.T.H. and O.K.F. gratefully acknowledge financial support by the U.S. DOE, Office of Science, Office of Basic Energy Sciences (grant No. DE-FG02 87ER13808) and Northwestern University. H.X. and W.A.G. were supported by NSF (EFRI-00155EI-1332411).

\section{REFERENCES}

(1) (a) Brédas, J.-L.; Beljonne, D.; Coropceanu, V.; Cornil, J. Chem. Rev. 2004, 104, 4971. (b) Kirner, S.; Sekita, M.; Guldi, D. M. Adv. Mater. 2014, 26, 1482.

(2) (a) Vura-Weis, J.; Abdelwahed, S. H.; Shukla, R.; Rathore, R.; Ratner, M. A.; Wasielewski, M. R. Science 2010, 328, 1547. (b) Goransson, E.; Emanuelsson, R.; Jorner, K.; Markle, T. F.; Hammarstrom, L.; Ottosson, H. Chem. Sci. 2013, 4, 3522. (c) Strauss, V.; Margraf, J. T.; Dirian, K.; Syrgiannis, Z.; Prato, M.; Wessendorf, C.; Hirsch, A.; Clark, T.; Guldi, D. M. Angew. Chem., Int. Ed. 2015, 54, 8292.

(3) (a) Aratani, N.; Kim, D.; Osuka, A. Acc. Chem. Res. 2009, 42, 1922. (b) Patwardhan, S.; Sengupta, S.; Siebbeles, L. D. A.; Würthner, F.; Grozema, F. C. J. Am. Chem. Soc. 2012, 134, 16147. (c) Parkinson, P.; Knappke, C. E. I.; Kamonsutthipaijit, N.; Sirithip, K.; Matichak, J. D.; Anderson, H. L.; Herz, L. M. J. Am. Chem. Soc. 2014, 136, 8217. (d) Ragoussi, M.-E.; Katsukis, G.; Roth, A.; Malig, J.; de la Torre, G.; Guldi, D. M.; Torres, T. J. Am. Chem. Soc. 2014, 136, 4593. (e) Sukegawa, J.; Schubert, C.; Zhu, X.; Tsuji, H.; Guldi, D. M.; Nakamura, E. Nat. Chem. 2014, 6, 899. (f) Rousseaux, S. A. L.; Gong, J. Q.; Haver, R.; Odell, B.; Claridge, T. D. W.; Herz, L. M.; Anderson, H. L. J. Am. Chem. Soc. 2015, 137, 12713. (g) Tanaka, T.; Osuka, A. Chem. Soc. Rev. 2015, 44, 943. (h) Antoniuk-Pablant, A.; Kodis, G.; Moore, A. L.; Moore, T. A.; Gust, D. J. Phys. Chem. B 2016, 120, 6687.

(4) Galindo, J. F.; Atas, E.; Altan, A.; Kuroda, D. G.; FernandezAlberti, S.; Tretiak, S.; Roitberg, A. E.; Kleiman, V. D. J. Am. Chem. Soc. 2015, 137, 11637.

(5) Ricks, A. B.; Brown, K. E.; Wenninger, M.; Karlen, S. D.; Berlin, Y. A.; Co, D. T.; Wasielewski, M. R. J. Am. Chem. Soc. 2012, 134, 4581.

(6) (a) Schmidt-Mende, L.; Fechtenkötter, A.; Müllen, K.; Moons, E.; Friend, R. H.; MacKenzie, J. D. Science 2001, 293, 1119. (b) Gunderson, V. L.; Smeigh, A. L.; Kim, C. H.; Co, D. T.; Wasielewski, M. R. J. Am. Chem. Soc. 2012, 134, 4363. (c) Young, R. M.; Dyar, S. M.; Barnes, J. C.; Juríček, M.; Stoddart, J. F.; Co, D. T.; Wasielewski, M. R. J. Phys. Chem. A 2013, 117, 12438. (d) Gallego, M.; Calbo, J.; Aragó, J.; Krick Calderon, R. M.; Liquido, F. H.; Iwamoto, T.; Greene, A. K.; Jackson, E. A.; Pérez, E. M.; Ortí, E.; Guldi, D. M.; Scott, L. T.; Martín, N. Angew. Chem., Int. Ed. 2014, 53, 2170. (e) Ryan, S. T. J.; Del Barrio, J.; Ghosh, I.; Biedermann, F.; Lazar, A. I.; Lan, Y.; Coulston, R. J.; Nau, W. M.; Scherman, O. A. J. Am. Chem. Soc. 2014, 136, 9053. (f) Ryan, S. T. J.; Young, R. M.; Henkelis, J. J.; Hafezi, N.; Vermeulen, N. A.; Hennig, A.; Dale, E. J.; Wu, Y.; Krzyaniak, M. D.; Fox, A.; Nau, W. M.; Wasielewski, M. R.; Stoddart, J. F.; Scherman, O. A. J. Am. Chem. Soc. 2015, 137, 15299.

(7) (a) Lee, C. Y.; Farha, O. K.; Hong, B. J.; Sarjeant, A. A.; Nguyen, S. T.; Hupp, J. T. J. Am. Chem. Soc. 2011, 133, 15858. (b) Oelsner, C.; Herrero, M. A.; Ehli, C.; Prato, M.; Guldi, D. M. J. Am. Chem. Soc. 2011, 133, 18696. (c) Jin, S.; Son, H.-J.; Farha, O. K.; Wiederrecht, G. P.; Hupp, J. T. J. Am. Chem. Soc. 2013, 135, 955. (d) Son, H.-J.; Jin, S.; Patwardhan, S.; Wezenberg, S. J.; Jeong, N. C.; So, M.; Wilmer, C. E.; Sarjeant, A. A.; Schatz, G. C.; Snurr, R. Q.; Farha, O. K.; Wiederrecht, G. P.; Hupp, J. T. J. Am. Chem. Soc. 2013, 135, 862. (e) Young, R. M.; 
Jensen, S. C.; Edme, K.; Wu, Y.; Krzyaniak, M. D.; Vermeulen, N. A.; Dale, E. J.; Stoddart, J. F.; Weiss, E. A.; Wasielewski, M. R.; Co, D. T. J. Am. Chem. Soc. 2016, 138, 6163.

(8) (a) Barnes, J. C.; Juríček, M.; Strutt, N. L.; Frasconi, M.; Sampath, S.; Giesener, M. A.; McGrier, P. L.; Bruns, C. J.; Stern, C. L.; Sarjeant, A. A.; Stoddart, J. F. J. Am. Chem. Soc. 2013, 135, 183. (b) Barnes, J. C.; Juríček, M.; Vermeulen, N. A.; Dale, E. J.; Stoddart, J. F. J. Org. Chem. 2013, 78, 11962. (c) Dale, E. J.; Vermeulen, N. A.; Juríček, M.; Barnes, J. C.; Young, R. M.; Wasielewski, M. R.; Stoddart, J. F. Acc. Chem. Res. 2016, 49, 262.

(9) Dyar, S. M.; Barnes, J. C.; Juríček, M.; Stoddart, J. F.; Co, D. T.; Young, R. M.; Wasielewski, M. R. Angew. Chem., Int. Ed. 2014, 53, 5371.

(10) (a) Slama-Schwok, A.; Jazwinski, J.; Béré, A.; MontenayGarestier, T.; Rougée, M.; Hélène, C.; Lehn, J.-M. Biochemistry 1989, 28, 3227. (b) Slama-Schwok, A.; Rougée, M.; Ibanez, V.; Geacintov, N. E.; Montenay-Garestier, T.; Lehn, J.-M.; Hélène, C. Biochemistry 1989, 28, 3234. (c) Brun, A. M.; Harriman, A. J. Am. Chem. Soc. 1991, 113, 8153. (d) Basuray, A. N.; Jacquot de Rouville, H.-P.; Hartlieb, K. J.; Kikuchi, T.; Strutt, N. L.; Bruns, C. J.; Ambrogio, M. W.; Avestro, A.-J.; Schneebeli, S. T.; Fahrenbach, A. C.; Stoddart, J. F. Angew. Chem., Int. Ed. 2012, 51, 11872. (e) Basuray, A. N.; de Rouville, H.-P. J.; Hartlieb, K. J.; Fahrenbach, A. C.; Stoddart, J. F. Chem. - Asian J. 2013, 8, 524. (f) Hartlieb, K. J.; Basuray, A. N.; Ke, C.; Sarjeant, A. A.; Jacquot de Rouville, H.-P.; Kikuchi, T.; Forgan, R. S.; Kurutz, J. W.; Stoddart, J. F. Asian J. Org. Chem. 2013, 2, 225. (g) Sampath, S.; Basuray, A. N.; Hartlieb, K. J.; Aytun, T.; Stupp, S. I.; Stoddart, J. F. Adv. Mater. 2013, 25, 2740. (h) Hartlieb, K. J.; Witus, L. S.; Ferris, D. P.; Basuray, A. N.; Algaradah, M. M.; Sarjeant, A. A.; Stern, C. L.; Nassar, M. S.; Botros, Y. Y.; Stoddart, J. F. ACS Nano 2015, 9, 1461. (i) Hou, X.; Ke, C.; Bruns, C. J.; McGonigal, P. R.; Pettman, R. B.; Stoddart, J. F. Nat. Commun. 2015, 6, 6884.

(11) Functionalized PDIs tend to exhibit increased solubilities in organic solvents. Although examples of the incorporation of a PDI unit, modified at its bay position (Figure 1a), into symmetrical cyclophanes have been report by the Würthner group, functionalization at the bay positions might interfere with binding of guests by the cyclophane, while functionalization at the imide positions with alkyl groups may not be appropriate in a robust cyclophane architecture. See: (a) Schlosser, F.; Moos, M.; Lambert, C.; Würthner, F. Adv. Mater. 2013, 25, 410. (b) Spenst, P.; Würthner, F. Angew. Chem., Int. Ed. 2015, 54, 10165.

(12) Hunter, C. A.; Sanders, J. K. M. J. Am. Chem. Soc. 1990, 112, 5525.

(13) In most cases, the geometry of molecules is not necessarily the same in solution as in the solid state because of thermal and environmental disorder. In the case of the DAPPBox ${ }^{4+}$, however, this potential mismatching of distances should be much less of an issue as a consequence of the rigidity of the cyclophane, where the range of accessible geometries in solution is quite limited. Therefore, it is not unreasonable to consider the crystal structure as an "equilibrium geometry" about which the solution-state geometry will fluctuate, as a result of both solvent and thermal fluctuations.

(14) Barnes, J. C.; Fahrenbach, A. C.; Dyar, S. M.; Frasconi, M.; Giesener, M. A.; Zhu, Z.; Liu, Z.; Hartlieb, K. J.; Carmieli, R.; Wasielewski, M. R.; Stoddart, J. F. Proc. Natl. Acad. Sci. U. S. A. 2012, 109, 11546.

(15) Takahashi, M.; Suzuki, Y.; Ichihashi, Y.; Yamashita, M.; Kawai, H. Tetrahedron Lett. 2007, 48, 357.

(16) Zheng, J.; Li, Y.; Sun, Y.; Yang, Y.; Ding, Y.; Lin, Y.; Yang, W. ACS Appl. Mater. Interfaces 2015, 7, 7241.

(17) Dolomanov, O. V.; Bourhis, L. J.; Gildea, R. J.; Howard, J. A. K.; Puschmann, H. J. Appl. Crystallogr. 2009, 42, 339.

(18) Sheldrick, G. Acta Crystallogr., Sect. C: Struct. Chem. 2015, 71, 3.

(19) Dixon, J. M.; Taniguchi, M.; Lindsey, J. S. Photochem. Photobiol.

2005, 81, 212. 